\title{
$\$$ Research Square

\section{Schistosomiasis, Upper Gastrointestinal Bleeding, and Health Related Quality of Life Measurements in Rural Africa}

Christopher Kenneth Opio ( $\sim$ opiokc@gmail.com )

Makerere University College of Health Sciences https://orcid.org/0000-0001-8898-6350

Francis Kazibwe

Bishop Stuart University

Lalitha Rejani

Makerere University College of Health Sciences

Narcis B Kabatereine

Vector control division, Ministry of Health Uganda

Ponsiano Ocama

Makerere University College of Health Sciences

\section{Research}

Keywords: Health related quality of life measurements, disability weights, upper gastrointestinal bleeding, schistosomiasis, EQ-5D/EQ-VAS

Posted Date: July 17th, 2020

DOl: https://doi.org/10.21203/rs.3.rs-42011/v1

License: (1) This work is licensed under a Creative Commons Attribution 4.0 International License.

Read Full License 


\section{Abstract}

Introduction Health related quality of life (HRQOL) measurements, which include disability weights, are important endpoints of health care delivery. These measurements are scarce for patients with upper gastrointestinal bleeding (UGIB) in rural sub-Saharan Africa (SSA) where schistosomiasis is endemic.

Methods and materials In 2014, we studied HRQOL measurements among patients with UGIB in SSA where schistosomiasis is endemic. Participants included adult inpatients and outpatients with a history of UGIB at a primary health facility. We measured HRQOL using the EuroQoL 5-dimension (EQ-VAS/EQ5D) instrument and derived disability weights from EQ-VAS and EQ5D measurements. We profiled each participant's medical history, physical examination, laboratory tests, imaging, and endoscopy findings. These were summarized through descriptive and inferential statistics.

Results We studied 107 participants with a median age of 45 years. All participants experienced $\geq 1$ lifetime episode of UGIB, $60 \%$ were females, while $22 \%$ had acute severe UGIB requiring admission, $98 \%$ hepatic schistosomiasis, $90 \%$ splenomegaly, $80 \%$ had esophageal varices, and $20 \%$ had peptic ulcers. Most $(80 \%)$ had ever received praziquantel for schistosomiasis. No participant had ever had an endoscopy for UGIB or received propranolol for prevention of recurrent variceal UGIB. Measures of the 5 dimensions of health revealed a large proportion of participants had problems related to self-care (76\%), anxiety/depression (88\%), and pain/discomfort (89\%). Few participants had problems with mobility(37\%). The mean/median EQ-VAS derived disability weights and EQ5D derived disability weights were $0.38 / 0.30$ and $0.38 / 0.34$, respectively. Participants with ascites, acute UGIB, and severe anemia were found to have the highest EQ-VAS and EQ5D median disability weights. At linear regression age, female gender, ascites, and acute UGIB predicted EQ-VAS derived disability weights $[F(4,100)=9.35, p<0.0000, R$ squared $=0.27]$ and $E Q 5 D$ derived disability weights $[F(4,100)=23.24, p<0.0000$, R-squared $=0.44]$. Within these models, all four factors were significantly predicted higher disability weights, P-value $<0.05$.

Conclusions In our study, older age, female gender, those with ascites or acute UGIB had the highest disability weights, and the greatest probability of having higher disability weights among patients with a history of UGIB where schistosomiasis is endemic. These findings are unique and improve the definitions of different health states among patients with UGIB and schistosomiasis.

\section{Introduction}

Upper gastrointestinal bleeding (UGIB) is a frequent cause of hospitalization and death in sub-Saharan Africa where S.mansoni is endemic. It often results in bleeding varices than bleeding peptic ulcer disease. The main causes of varices in sub-Saharan Africa include periportal fibrosis due to S.mansoni infection and liver cirrhosis (hepatitis B/C and alcoholic liver disease)[1-4]. Upper gastrointestinal bleeding among patients with varices is a medical emergency that usually presents with hematemesis, melena, hypotension, severe anemia, and sometimes recurrent variceal hemorrhage[5]. In chronic schistosomiasis, recurrent variceal hemorrhage usually occurs within 6 weeks of hospitalization after the initial episode of 
acute variceal hemorrhage and is associated with a high risk of death. It is shown one out of ten persons with UGIB due to chronic schistosomiasis will die within 6 weeks of acute variceal hemorrhage and 3 out of 10 persons will re-bleed over the same period[6]. Early diagnosis and appropriate preventive therapy are therefore recommended for those who experience variceal UGIB due to schistosomiasis. This has been shown to mitigate disease progression, prevent new acute episodes of UGIB, and decrease hospital admissions[7-9]. However, the impact of these interventions remains elusive due to the paucity of baseline or follow-up data on measures of health-related quality of life (HRQOL) from this patient population. Health related quality of life measurements are important endpoints of health care delivery. Health related quality of life measurements are surrogate markers of disability or health loss or disease impact, and can be used to evaluate treatment response[10,11].

Few studies from brazil and china have described health losses among patients with chronic schistosomiasis. These studies showed increased disability among those with severe periportal fibrosis or advanced disease $[7,12,13]$.

On the other hand, data about health-related quality of life measurements about chronic schistosomiasis and/or related complications like UGIB and ascites is scarce [14]. We studied health related quality of life measurements among patients with UGIB at a rural primary health facility in sub-Saharan Africa where $S$. mansoni is endemic.

\section{Materials And Methods}

This was a cross sectional study that involved individuals attending the outpatient department (OPD) of Pakwach health centre IV and inpatients admitted with acute severe UGIB at the same health facility. Pakwach health centre is a rural primary health care facility located at the banks of the Albert Nile in a region that is a hot spot for S.mansoni infection. Pakwach health centre provides outpatient and inpatient health care services to a population who are mainly fisherpersons and/or farmers. Epidemiological data suggest close to $50 \%$ of the populations served by this facility are actively infected with S.mansoni despite over a decade of Praziquantel chemotherapy. Medical records from the facility indicated about 120 patients every year are admitted for acute UGIB attributable to chronic schistosomiasis. Eligibility for study participation included written informed consent and ascent, all patients $\geq 12$ years of age with a medical history of UGIB (past or current). We excluded pregnant women, HIV positive individuals, and any participant unable to have endoscopy for UGIB. Upper gastrointestinal bleeding was defined by any lifetime history of hematemesis, melena, or hematochezia.

Participants at the OPD were systematically enrolled while inpatients were consecutively recruited over 6 weeks. A detailed medical history that included socio-demographic data, exposure to schistosoma species or alcohol, treatment of schistosomiasis and time from the last treatment, history of UGIB, and other relevant past medical history. Participants were examined for stigmata of chronic liver disease, and vital signs[15, 16]. 
Measures of health-related quality of life were obtained using the three-level European Quality of Life 5Dimensions (EQ-5D-Y) questionnaire and the visual analogue scale (EQ-VAS) from the EuroQol Group $\left(E Q-5 D^{T M}\right)$.

The EQ-5D-Y is a multi-attribute utility instrument. The EQ-5D represents measures of personal well-being. It has 5 dimensions that include mobility, self-care, usual activities, pain/discomfort, and anxiety/depression. Each dimension is scored as three levels: no problems (level 1), some problems (level 2), and extreme problems (level 3). The EQ-5D data was converted into an index of health (EQ5D index) using crosswalk values from Zimbabwe. The index represents a measure of health from 0 for death to 1 for perfect health. The EQ-VAS is a scale that varies from 0 , the worst imaginable health state, to 100 , the best imaginable health state. The EQ-VAS asks patients to indicate their overall health on a vertical visual analogue scale( 0 to 100$)$.

The EQ-VAS and EQ5D index were then transformed to disability weights (DW) by the formulas [ EQVAS$D W=1-(V A S / 100)]$ and [EQ5D index $-D W=1-E Q 5 D$ index] respectively. $A$ disability weight is a weight factor that reflects the severity of the disease on a scale from 0 (perfect health) to 1 (equivalent to death). Disability weights characterize the amount of health loss associated with specific health outcomes, and are used to calculate years lived with disability[12, 17-19].

Blood was analyzed generating 3-part hematology indices (using a compact Sysmex KX-21 hematology analyzer), hepatitis B and C viral blood serology results (obtained from commercially available rapid diagnostic test kits), and malaria antigen test results (from rapid diagnostic test kits). Stool microscopy was performed for ova and urine for schistosomiasis using the urine circulating cathodic antigen (CCA) test by Rapid diagnostics. Trans-abdominal ultrasonography performed by a trained sonographer according to the modified World Health Organization Niamey protocol using the SONOSTAR model SS8, a portable ultrasound with a $3.5 \mathrm{MHz}$ convex probe. Upper digestive endoscopy was performed using a Pentax EPKi digital video processor and a Pentax $9.8 \mathrm{~mm}$ video gastroscope after a local anesthetic (Xylocaine spray) by a gastroenterologist.

Data was transcribed from questionnaires and later entered into a Microsoft Access database. This was edited to ensure quality and exported to Stata version 16 (STATA Corp, Lakeway, College Station, Texas, USA). Descriptive and inferential statistics were generated describing the study population and measures of HRQOL (EQVAS, EQ5D, EQ5D index, EQVAS-DW, and EQ5D index-DW), and factors associated with EQVAS-DW, and EQ5D index-DW. Other variables were designated covariates. Categorical data were summarized as proportions with standard errors (95\% confidence intervals). Continuous data were summarized as means, medians, standard deviations (SD), and $95 \%$ confidence intervals. However, interquartile ranges were also generated for some medians. We generated a number of linear regression models describing the association between potential independent variables and the two separate dependent outcomes (EQVAS-DW and EQ5D index-DW). Selection of appropriate covariates and the best model was guided by background knowledge, significance criterion, information criteria, and penalized likelihood methods in Stata. We presented standardized beta coefficients for the best models with their 
standard errors and $p$-values. These were reported as percentage points ( percentage points $=$ beta coefficient $x$ 100). A significance level ( $p$-value $<0.05$ ) was considered, and confidence intervals or standard errors supported inference. These results were summarized as text, in tables, and as figures.

\section{Ethics statement}

This was a routine cross-sectional study that involved human participants. It was approved by School of Medicine, Makerere University, Institutional review board, Kampala, Uganda (\#REC REF2011-244), and the Uganda National Council for Science and Technology, Kampala, Uganda (UNCST approval \#, HS 1620). The study was conducted according to the principles expressed in the Declaration of Helsinki. Written informed consent was obtained from all participants.

\section{Results}

The study was conducted over 6 weeks between the months of July and August 2014.

All participants had a past or current medical history of UGIB. One fifth (23 participants out of 107 enrolled) presented as medical emergencies and were admitted with acute severe upper gastrointestinal bleeding over the study period. We screened 324 at the outpatient's department over the same period and were able to enroll 84 patients with a past medical history of UGIB. The youngest was 25 years and the oldest 71 years. The median age was 45 years, interquartile range of 13 years. The female-male ratio was 3 to 2. Most participants were long-term residents of Pakwach. All participants had frequent contact with the waters of the Nile and nearly all were either fisherpersons or farmers by occupation. Among our participants, $54 \%$ had a prior diagnosis of intestinal schistosomiasis, $88 \%$ had ever received Praziquantel in the past, $8 \%$ had active infection at the time of enrolment, $90 \%$ had splenomegaly on physical exam, and $96 \%$ had periportal fibrosis and/or cirrhosis at ultrasonography. All reported experiencing UGIB with $96 \%$ reporting at least one lifetime episode of hematemesis, and $96 \%$ reporting a past admission for UGIB. No participant has ever had an endoscopy for UGIB nor was on propranolol for prevention of recurrent variceal bleeding. Endoscopy was performed on all 107 participants during the study. Following endoscopy, we found 86 had varices, 8 had both varices and peptic ulcers, and 21 had peptic ulcers alone.

\section{EQ5D-Y, EQ- VAS, and derived disability weights}

Measures of the 5 dimensions of health revealed $80(76 \%)$ participants had some or extreme problems in self-care, $92(88 \%)$ participants reported some or extreme anxiety or depression, and 93(89\%) participants reported experiencing some or extreme pain or discomfort. Only 39(37\%) participants reported some or extreme problems in their mobility. However, $48(46 \%)$ participants were able to undertake their usual activities without any problems, Fig. 1. 
The median health index for 105 available participant records was 0.66 with IQR $=0.102$ and the mean index was $0.62, S D=21$. The median visual analogue score (VAS) was for 105 available participants, records were $70, I Q R=30$, and mean VAS of $62, S D=21$.

The median VAS disability weights [EQVAS-DW $=1-(\mathrm{VAS} / 100)$ ] was $0.3, \mathrm{IQR}=0.3$ and mean VAS disability weights [EQVAS-DW $=1-(V A S / 100)$ ] was $0.38, S D=0.23$. The median EQ-5D disability weights [ EQ5D index-DW $=1-E Q 5 D$ index $]$ and mean EQ-5d disability weights [ EQ5D index-DW = 1-EQ5D index] were 0.34, $\mathrm{IQR}=0.10$, and $0.38, \mathrm{SD}=0.21$ respectively.

\section{Multiple regression of factors associated with EQVAS-DW and EQ5D index-DW)}

Multiple linear regression revealed age, female gender, ascites, and acute UGIB predicted EQ-VAS derived disability weights $[F(4,100)=9.35, p<0.0000$, R-squared $=0.27]$ and $E Q 5 D$ derived disability weights $[F(4,100)=23.24, p<0.0000$, R-squared $=0.44]$. Within these two best models, all five factors were significantly associated with higher disability weights, Table 3 . Put simply, the probability of having a higher EQ5D-DW was increased by 0.5 percentage points for every year lived, 12 percentage points if one was female, 13 percentage points if diagnosed with ascites, and 13 percentage points if one experienced acute UGIB. While the probability of having a higher EQVAS-DW was increased by 0.4 percentage points for every year lived, 7 percentage points if one was female, 15 percentage points if diagnosed with ascites on ultrasound, and 28 percentage points if one experienced acute UGIB, Table 3. 
Table 1

Description of the study population (frequencies, proportions, and estimates)

\begin{tabular}{|c|c|c|c|}
\hline Variable & $\mathbf{N}$ & Proportion or Median(IQR) & {$[95 \% \mathrm{Cl}]$} \\
\hline Age & 107 & 45 years (13 years) & $43-49$ years \\
\hline Female & 107 & $60 \%$ & $50-69 \%$ \\
\hline Stayed in Pakwach $>10$ years & 107 & $88 \%$ & $80-93 \%$ \\
\hline River contact $\geq 5-7$ times/week & 107 & $99 \%$ & $94-100 \%$ \\
\hline Farmer & 107 & $74 \%$ & $65-81 \%$ \\
\hline History of blood stool (hematochezia) & 107 & $51 \%$ & $42-61 \%$ \\
\hline History of vomited blood ( hematemesis) & 107 & $96 \%$ & $90-99 \%$ \\
\hline History of black stool (melena) & 107 & $44 \%$ & $35-54 \%$ \\
\hline Past diagnosis schistosomiasis( stool) & 107 & $54 \%$ & $45-63 \%$ \\
\hline Past use of Praziquantel & 107 & $88 \%$ & $80-93 \%$ \\
\hline Toilet or pit latrine & 107 & $99 \%$ & $94-100 \%$ \\
\hline Admitted for UGIB & 107 & $96 \%$ & $90-99 \%$ \\
\hline Blood transfusion & 107 & $68 \%$ & $59-76 \%$ \\
\hline Acute severe UGIB & 107 & $22 \%$ & $15-30 \%$ \\
\hline Splenomegaly (palpable spleen)* & 102 & $90 \%$ & $83-95 \%$ \\
\hline Ascites & 107 & $17 \%$ & $11-25 \%$ \\
\hline Jaundice & 107 & $10 \%$ & $8-18 \%$ \\
\hline Oedema & 107 & $11 \%$ & $6-19 \%$ \\
\hline Flap & 107 & $9 \%$ & $5-17 \%$ \\
\hline Spleen size ** & 105 & $15.8 \mathrm{~cm}(4.1 \mathrm{~cm})$ & $14.6-16.3 \mathrm{~cm}$ \\
\hline EQ-VAS *** & 105 & $70(30)$ & $60-70$ \\
\hline
\end{tabular}

UGIB - upper gastrointestinal bleeding, CCA - cathodic antigen test, $\mathrm{HCV}$ - hepatitis C virus, HBsAg hepatitis B surface antigen, VAS - visual analogue score, IQR - interquartile range.

*- spleen could not be palpated because of tense ascites for 5 participants.

** ultrasound evaluation could not be completed because of tense ascites for 2 participants.

*** Incomplete EQ-VAS and EQ5D-index for 2 participants.

\# Stool was not performed for 1 participant. 


\begin{tabular}{|llll|}
\hline Variable & N & Proportion or Median(IQR) & [95\% Cl] \\
\hline Stool ova for S.mansoni at enrollment \# & 106 & $7 \%$ & $3-13 \%$ \\
\hline Urine CCA test for S.mansoni at enrollment & 107 & $8 \%$ & $4-16 \%$ \\
\hline Anti -HCV Ab positive & 107 & $26 \%$ & $19-35 \%$ \\
\hline HBsAg positive & 107 & $7 \%$ & $3-13 \%$ \\
\hline Portal vein diameter $\geq 13$ mm & 107 & $74 \%$ & $65-81 \%$ \\
\hline Varices at endoscopy & 107 & $80 \%$ & $72-87 \%$ \\
\hline A( No fibrosis) & 107 & $4 \%$ & $1-10 \%$ \\
\hline C( mild periportal fibrosis) & 107 & $5 \%$ & $2-11 \%$ \\
\hline D( moderate periportal fibrosis) & 107 & $32 \%$ & $24-41 \%$ \\
\hline E ( Severe periportal fibrosis) & 107 & $20 \%$ & $13-28 \%$ \\
\hline F ( Severe periportal fibrosis) & 107 & $4 \%$ & $1-10 \%$ \\
\hline X ( Liver cirrhosis like picture) & 107 & $37 \%$ & $28-46 \%$ \\
\hline EQD5-Index (Zimbabwe)*** & 105 & 0.66 (0.10) & $0.652-0.695$ \\
\hline UGIB - upper gastrointestinal bleeding, CCA - cathodic antigen test, HCV - hepatitis C virus, HBsAg - & \\
\hline hepatitis B surface antigen, VAS - visual analogue score, IQR - interquartile range. & \\
\hline *- spleen could not be palpated because of tense ascites for 5 participants. & \\
\hline ** ultrasound evaluation could not be completed because of tense ascites for 2 participants. \\
\hline *** Incomplete EQ-VAS and EQ5D-index for 2 participants. & & & \\
\hline \# Stool was not performed for 1 participant. & & & \\
\hline
\end{tabular}


Table 2

Descriptive statistics about EQ-VAS and EQ5D derived disability weights.

\begin{tabular}{|c|c|c|c|c|c|}
\hline & & EQVAS-DW & $\begin{array}{l}\text { EQVAS- } \\
\text { DW }\end{array}$ & $\begin{array}{l}\text { EQ5D } \\
\text { index-DW }\end{array}$ & $\begin{array}{l}\text { EQ5D } \\
\text { index-DW }\end{array}$ \\
\hline & $\mathrm{n}$ & Mean & Median & Mean & Median \\
\hline & & $(95 \% \mathrm{Cl})$ & $(95 \% \mathrm{Cl})$ & $(95 \% \mathrm{Cl})$ & $(95 \% \mathrm{Cl})$ \\
\hline \multirow[t]{2}{*}{ All participants } & 105 & 0.38 & 0.30 & 0.38 & 0.34 \\
\hline & & $(0.34,0.43)$ & $\begin{array}{l}(0.30 \\
0.40)\end{array}$ & $\begin{array}{l}(0.34 \\
0.43)\end{array}$ & $\begin{array}{l}(0.31 \\
0.35)\end{array}$ \\
\hline \multirow[t]{2}{*}{ Age $<40$ years } & 29 & 0.32 & 0.30 & 0.32 & 0.31 \\
\hline & & $(0.23,0.36)$ & $\begin{array}{l}(0.20 \\
0.30)\end{array}$ & $\begin{array}{l}(0.27 \\
0.38)\end{array}$ & $\begin{array}{l}(0.29 \\
0.35)\end{array}$ \\
\hline \multirow[t]{2}{*}{ Age $\geq 40$ years } & 76 & 0.42 & 0.40 & 0.41 & 0.35 \\
\hline & & $(0.37,0.47)$ & $\begin{array}{l}(0.30 \\
0.50)\end{array}$ & $\begin{array}{l}(0.36 \\
0.46)\end{array}$ & $\begin{array}{l}(0.31, \\
0.40)\end{array}$ \\
\hline \multirow[t]{2}{*}{ Female } & 64 & 0.40 & 0.35 & 0.42 & 0.35 \\
\hline & & $(0.35,0.46)$ & $\begin{array}{l}(0.30 \\
0.50)\end{array}$ & $\begin{array}{l}(0.36 \\
0.48)\end{array}$ & $\begin{array}{l}(0.31 \\
0.40)\end{array}$ \\
\hline \multirow[t]{2}{*}{ Male } & 41 & 0.35 & 0.40 & 0.32 & 0.40 \\
\hline & & $(0.29,0.42)$ & $\begin{array}{l}(0.30 \\
0.50)\end{array}$ & $\begin{array}{l}(0.28 \\
0.37)\end{array}$ & $\begin{array}{l}(0.30 \\
0.50)\end{array}$ \\
\hline \multirow[t]{2}{*}{ Occupation, other } & 20 & 0.44 & 0.45 & 0.32 & 0.31 \\
\hline & & $(0.33,0.55)$ & $\begin{array}{l}(0.20 \\
0.60)\end{array}$ & $\begin{array}{l}(0.24, \\
0.41)\end{array}$ & $\begin{array}{l}(0.21, \\
0.40)\end{array}$ \\
\hline Occupation, & 6 & 0.33 & 0.25 & 0.32 & 0.30 \\
\hline fishing only & & $(0.15,0.52)$ & $\begin{array}{l}(0.11, \\
0.68)\end{array}$ & $\begin{array}{l}(0.21, \\
0.43)\end{array}$ & $\begin{array}{l}(0.15, \\
0.52)\end{array}$ \\
\hline Occupation, & 70 & 0.38 & 0.35 & 0.41 & 0.35 \\
\hline farming only & & $(0.33,0.43)$ & $\begin{array}{l}(0.30 \\
0.40)\end{array}$ & $\begin{array}{l}(0.37 \\
0.46)\end{array}$ & $\begin{array}{l}(0.31 \\
0.40)\end{array}$ \\
\hline Occupation, & 9 & 0.34 & 0.30 & 0.36 & 0.35 \\
\hline farming and fishing & & $(0.25,0.44)$ & $\begin{array}{l}(0.21 \\
0.50)\end{array}$ & $\begin{array}{l}(0.25 \\
0.47)\end{array}$ & $\begin{array}{l}(0.22, \\
0.48)\end{array}$ \\
\hline
\end{tabular}

UGIB - upper gastrointestinal bleeding, BMI - body mass index, WHO-world health organization. 


\begin{tabular}{|c|c|c|c|c|c|}
\hline & & EQVAS-DW & $\begin{array}{l}\text { EQVAS- } \\
\text { DW }\end{array}$ & $\begin{array}{l}\text { EQ5D } \\
\text { index-DW }\end{array}$ & $\begin{array}{l}\text { EQ5D } \\
\text { index-DW }\end{array}$ \\
\hline 5-7 times weekly & 104 & 0.39 & 0.30 & 0.38 & 0.33 \\
\hline contact with River Nile & & $(0.34,0.43)$ & $\begin{array}{l}(0.30 \\
0.40)\end{array}$ & $\begin{array}{l}(0.34 \\
0.43)\end{array}$ & $\begin{array}{l}(0.31 \\
0.35)\end{array}$ \\
\hline $\begin{array}{l}\text { 3-4 times weekly } \\
\text { contact with River Nile }\end{array}$ & 1 & 0.2 & 0.2 & 0.35 & 0.35 \\
\hline \multicolumn{6}{|c|}{ History of S.mansoni stool ova } \\
\hline \multirow[t]{2}{*}{ Yes } & 57 & 0.41 & 0.4 & 0.39 & 0.35 \\
\hline & & $(0.35,0.48)$ & $(0.3,0.5)$ & $(0.32,0.46)$ & $(0.31,0.38)$ \\
\hline \multirow[t]{2}{*}{ No } & 48 & 0.35 & 0.30 & 0.37 & 0.33 \\
\hline & & $(0.30,0.40)$ & $(0.30,0.5)$ & $(0.33,0.42)$ & $(0.31,0.37)$ \\
\hline \multicolumn{6}{|l|}{$\begin{array}{l}\text { History of praziquantel } \\
\text { chemotherapy }\end{array}$} \\
\hline \multirow[t]{2}{*}{ Yes } & 93 & 0.40 & 0.40 & 0.40 & 0.35 \\
\hline & & $(0.36,0.45)$ & $\begin{array}{l}(0.30 \\
0.50)\end{array}$ & $\begin{array}{l}(0.35 \\
0.44)\end{array}$ & $\begin{array}{l}(0.31 \\
0.37)\end{array}$ \\
\hline \multirow[t]{2}{*}{ No } & 12 & 0.23 & 0.20 & 0.28 & 0.31 \\
\hline & & $(0.14,0.33)$ & $\begin{array}{l}(0.10, \\
0.30)\end{array}$ & $\begin{array}{l}(0.24, \\
0.33)\end{array}$ & $(0.22,0.34)$ \\
\hline \multicolumn{6}{|l|}{ Past symptoms of UGIB } \\
\hline \multirow[t]{2}{*}{ Melena only } & 2 & 0.70 & 0.70 & 0.50 & 0.50 \\
\hline & & & $(0.7,0.70)$ & $(0.45,0.56)$ & $(0.48,0.53)$ \\
\hline \multirow[t]{2}{*}{ Hematmemesis only } & 25 & 0.28 & 0.30 & 0.36 & 0.31 \\
\hline & & $(0.2 v, 0.00)$ & $\begin{array}{l}(0.20 \\
0.30)\end{array}$ & $(0.29,0.44)$ & $(0.30,0.35)$ \\
\hline \multirow[t]{2}{*}{ Hematemesis and melena } & 24 & 0.30 & 0.30 & 0.35 & 0.35 \\
\hline & & $(0.23,0.38)$ & $\begin{array}{l}(0.20 \\
0.40)\end{array}$ & $(0.29,0.42)$ & $(0.27,0.40)$ \\
\hline \multirow[t]{2}{*}{ Hematochesia only } & 1 & 0.2 & 0.20 & 0.31 & 0.31 \\
\hline & & & $\begin{array}{l}(0.20 \\
0.20)\end{array}$ & & $(0.31,0.31)$ \\
\hline
\end{tabular}

UGIB - upper gastrointestinal bleeding, BMI - body mass index, WHO-world health organization. 


\begin{tabular}{|c|c|c|c|c|c|}
\hline & & EQVAS-DW & $\begin{array}{l}\text { EQVAS- } \\
\text { DW }\end{array}$ & $\begin{array}{l}\text { EQ5D } \\
\text { index-DW }\end{array}$ & $\begin{array}{l}\text { EQ5D } \\
\text { index-DW }\end{array}$ \\
\hline \multirow[t]{2}{*}{ Hematochesia and melana } & 1 & 0.1 & 0.10 & 0.28 & 0.28 \\
\hline & & & $\begin{array}{l}(0.10 \\
0.10)\end{array}$ & & $(0.28,0.28)$ \\
\hline \multirow[t]{2}{*}{ Hematochesia and hematemesis } & 32 & 0.40 & 0.35 & 0.35 & 0.31 \\
\hline & & $(0.33,0.46)$ & $\begin{array}{l}(0.30 \\
0.50)\end{array}$ & $(0.28,0.43)$ & $(0.31,0.35)$ \\
\hline \multirow{2}{*}{$\begin{array}{l}\text { Hematemesis, melena, and } \\
\text { hematochezia }\end{array}$} & 20 & 0.58 & 0.60 & 0.50 & 0.40 \\
\hline & & $(0.47,0.68)$ & $\begin{array}{l}(0.41 \\
0.70)\end{array}$ & $(0.38,0.62)$ & $(0.31,0.76)$ \\
\hline \multicolumn{6}{|l|}{ Past admission for UGIB } \\
\hline \multirow[t]{2}{*}{ Yes } & 101 & 0.39 & 0.30 & 0.39 & 0.35 \\
\hline & & $(0.21,0.35)$ & $\begin{array}{l}(0.30 \\
0.40)\end{array}$ & $(0.35,0.43)$ & $(0.31,0.35)$ \\
\hline \multirow[t]{2}{*}{ No } & 4 & 0.20 & 0.20 & 0.28 & 0.29 \\
\hline & & $(0.10,0.32)$ & $\begin{array}{l}(0.10 \\
0.30)\end{array}$ & $(0.21,0.35)$ & $(0.21,0.31)$ \\
\hline \multicolumn{6}{|l|}{ History of blood transfusion } \\
\hline \multirow[t]{2}{*}{ Yes } & 72 & 0.42 & 0.40 & 0.40 & 0.35 \\
\hline & & $(0.37,0.47)$ & $\begin{array}{l}(0.30 \\
0.50)\end{array}$ & $(0.35,0.46)$ & $(0.31,0.40)$ \\
\hline \multirow[t]{2}{*}{ No } & 33 & 0.31 & 0.3 & 0.35 & 0.31 \\
\hline & & $(0.25,0.36)$ & $\begin{array}{l}(0.20 \\
0.30)\end{array}$ & $(0.29,0.41)$ & $(0.31,0.35)$ \\
\hline \multicolumn{6}{|l|}{ Splenomegaly on Palpation } \\
\hline \multirow[t]{2}{*}{ Yes } & 91 & 0.37 & 0.30 & 0.37 & 0.32 \\
\hline & & $(0.33,0.42)$ & $(0.3,0.40)$ & $\begin{array}{l}(0.33, \\
0.40)\end{array}$ & $(0.31,0.35)$ \\
\hline \multirow[t]{2}{*}{ No } & 10 & 0.35 & 0.30 & 0.45 & 0.31 \\
\hline & & $(0.21,0.49)$ & $\begin{array}{l}(0.20, \\
0.50)\end{array}$ & $(0.25,0.64)$ & $(0.25,0.75)$ \\
\hline
\end{tabular}

UGIB - upper gastrointestinal bleeding, BMI - body mass index, WHO-world health organization. 


\begin{tabular}{|c|c|c|c|c|c|}
\hline & & EQVAS-DW & $\begin{array}{l}\text { EQVAS- } \\
\text { DW }\end{array}$ & $\begin{array}{l}\text { EQ5D } \\
\text { index-DW }\end{array}$ & $\begin{array}{l}\text { EQ5D } \\
\text { index-DW }\end{array}$ \\
\hline \multirow{2}{*}{$\begin{array}{l}\text { Difficult to assess due to tense } \\
\text { ascites }\end{array}$} & \multirow[t]{2}{*}{4} & 0.73 & 0.70 & 0.66 & 0.51 \\
\hline & & $(0.53,0.93)$ & $\begin{array}{l}(0.60 \\
0.90)\end{array}$ & $(0.14,1.2)$ & $(0.48,1.15)$ \\
\hline \multicolumn{6}{|l|}{ Ascites ( clinical exam) } \\
\hline \multirow[t]{2}{*}{ Yes } & \multirow[t]{2}{*}{17} & 0.52 & 0.5 & 0.51 & 0.42 \\
\hline & & $(0.42,0.62)$ & $\begin{array}{l}(0.40 \\
0.70)\end{array}$ & $(0.39,0.62)$ & $(0.38,0.55)$ \\
\hline \multirow[t]{2}{*}{ No } & \multirow[t]{2}{*}{88} & 0.37 & 0.30 & 0.36 & 0.31 \\
\hline & & $(0.31,0.40)$ & $\begin{array}{l}(0.30 \\
0.40)\end{array}$ & $(0.32,0.40)$ & $(0.31,0.35)$ \\
\hline \multicolumn{6}{|l|}{ Jaundice } \\
\hline \multirow[t]{2}{*}{ Yes } & \multirow[t]{2}{*}{11} & 0.43 & 0.40 & 0.44 & 0.40 \\
\hline & & $(0.31,0.54)$ & $\begin{array}{l}(0.30 \\
0.53)\end{array}$ & $\begin{array}{l}(0.33, \\
0.56)\end{array}$ & $(0.35,0.65)$ \\
\hline \multirow[t]{2}{*}{ No } & \multirow[t]{2}{*}{94} & 0.38 & 0.30 & 0.38 & 0.31 \\
\hline & & $(0.33,0.42)$ & $\begin{array}{l}(0.30 \\
0.40)\end{array}$ & $(0.33,0.42)$ & $(0.31,0.35)$ \\
\hline \multicolumn{6}{|l|}{ Edema } \\
\hline \multirow[t]{2}{*}{ Yes } & \multirow[t]{2}{*}{11} & 0.59 & 0.60 & 0.56 & 0.48 \\
\hline & & $(0.46,0.72)$ & $\begin{array}{l}(0.50 \\
0.70)\end{array}$ & $(0.37,0.74)$ & $(0.34,0.77)$ \\
\hline \multirow[t]{2}{*}{ No } & \multirow[t]{2}{*}{94} & 0.36 & 0.30 & 0.36 & 0.31 \\
\hline & & $(0.32,0.40)$ & $\begin{array}{l}(0.30 \\
0.40)\end{array}$ & $(0.32,0.40)$ & $(0.31,0.35)$ \\
\hline \multicolumn{6}{|l|}{$\mathrm{BMI}+/$ - ascites } \\
\hline$<18.5$ and ascites & 1 & 0.7 & 0.7 & 0.77 & 0.77 \\
\hline \multirow[t]{2}{*}{$<18.5$, no ascites } & \multirow[t]{2}{*}{18} & 0.45 & 0.40 & 0.51 & 0.40 \\
\hline & & $(0.30,0.56)$ & $\begin{array}{l}(0.30 \\
0.50)\end{array}$ & $(0.36,0.67)$ & $(0.30,0.80)$ \\
\hline
\end{tabular}

UGIB - upper gastrointestinal bleeding, BMI - body mass index, WHO -world health organization. 


\begin{tabular}{|c|c|c|c|c|c|}
\hline & & EQVAS-DW & $\begin{array}{l}\text { EQVAS- } \\
\text { DW }\end{array}$ & $\begin{array}{l}\text { EQ5D } \\
\text { index-DW }\end{array}$ & $\begin{array}{l}\text { EQ5D } \\
\text { index-DW }\end{array}$ \\
\hline \multirow[t]{2}{*}{$18.5-25$ and ascites } & 16 & 0.51 & 0.5 & 0.49 & 0.41 \\
\hline & & $(0.41,0.62)$ & $\begin{array}{l}(0.35 \\
0.65)\end{array}$ & $(0.37,0.61)$ & $(0.36,0.54)$ \\
\hline \multirow[t]{2}{*}{$18.5-25$, no ascites } & 64 & 0.33 & 0.30 & 0.32 & 0.31 \\
\hline & & $(0.28,0.38)$ & $\begin{array}{l}(0.20 \\
0.30)\end{array}$ & $(0.28,0.35)$ & $(0.31,0.34)$ \\
\hline$>25$ and ascites & 0 & 0 & 0 & 0 & 0 \\
\hline \multirow[t]{2}{*}{$>25$, no ascites } & 6 & 0.40 & 0.3 & 0.39 & 0.33 \\
\hline & & $(0.17,0.63)$ & $\begin{array}{l}(0.21 \\
0.78)\end{array}$ & $(0.19,0.56)$ & $(0.27,0.72)$ \\
\hline \multicolumn{6}{|l|}{ Esophageal varices } \\
\hline \multirow[t]{2}{*}{ Fo-no varices } & 21 & 0.35 & 0.30 & 0.35 & 0.31 \\
\hline & & $(0.26,0.44)$ & $\begin{array}{l}(0.20 \\
0.46)\end{array}$ & $(0.27,0.43)$ & $(0.31,0.38)$ \\
\hline \multirow[t]{2}{*}{ F1-small varices } & 12 & 0.33 & 0.25 & 0.34 & 0.31 \\
\hline & & $(0.20,0.45)$ & $(0.20,0.5)$ & $(0.21,0.48)$ & $(0.21,0.42)$ \\
\hline \multirow[t]{2}{*}{ F2- medium varices } & 32 & 0.34 & 0.30 & 0.37 & 0.31 \\
\hline & & $(0.28,0.40)$ & $\begin{array}{l}(0.20 \\
0.40)\end{array}$ & $(0.30,0.44)$ & $(0.20,0.40)$ \\
\hline \multirow[t]{2}{*}{ F3- large varices } & 40 & 0.46 & 0.45 & 0.42 & 0.35 \\
\hline & & $(0.38,0.53)$ & $(0.30,0.50)$ & $(0.35,0.50)$ & $(0.31,0.40)$ \\
\hline \multicolumn{6}{|c|}{ WHO anemia grading $(\mathrm{g} / \mathrm{dL})$} \\
\hline \multirow[t]{2}{*}{0 to 7.9} & 32 & 0.49 & 0.50 & 0.49 & 0.40 \\
\hline & & $(0.42,0.56)$ & $\begin{array}{l}(0.40 \\
0.50)\end{array}$ & $(0.41,0.56)$ & $(0.40,0.50)$ \\
\hline \multirow[t]{2}{*}{8 to 10.9} & 31 & 0.37 & 0.40 & 0.37 & 0.31 \\
\hline & & $(0.31,0.44)$ & $(0.20,0.50)$ & $(0.29,0.46)$ & $(0.30,0.38)$ \\
\hline \multirow[t]{2}{*}{11 to 11.9} & 16 & 0.35 & 0.30 & 0.38 & 0.31 \\
\hline & & $(0.23,0.47)$ & $\begin{array}{l}(0.15 \\
0.50)\end{array}$ & $(0.26,0.49)$ & $(0.30,0.38)$ \\
\hline
\end{tabular}

UGIB - upper gastrointestinal bleeding, BMI - body mass index, WHO-world health organization. 


\begin{tabular}{|c|c|c|c|c|c|}
\hline & & EQVAS-DW & $\begin{array}{l}\text { EQVAS- } \\
\text { DW }\end{array}$ & $\begin{array}{l}\text { EQ5D } \\
\text { index-DW }\end{array}$ & $\begin{array}{l}\text { EQ5D } \\
\text { index-DW }\end{array}$ \\
\hline \multirow[t]{2}{*}{$\geq 12$} & 26 & 0.29 & 0.21 & 0.28 & 0.31 \\
\hline & & $(0.20,0.37)$ & $\begin{array}{l}(0.30 \\
0.38)\end{array}$ & $(0.23,0.33)$ & $(0.22,0.31)$ \\
\hline \multicolumn{6}{|l|}{ Acute UGIB } \\
\hline \multirow[t]{2}{*}{ Yes } & 22 & 0.62 & 0.60 & 0.51 & 0.45 \\
\hline & & $(0.54,0.70)$ & $(0.5,0.70)$ & $(0.42,0.60)$ & $(0.40,0.63)$ \\
\hline \multirow[t]{2}{*}{ No } & 83 & 0.32 & 0.30 & 0.35 & 0.31 \\
\hline & & $(0.28,0.36)$ & $\begin{array}{l}(0.20 \\
0.40)\end{array}$ & $(0.31,0.39)$ & $(0.31,0.35)$ \\
\hline \multirow[t]{2}{*}{ No acute UGIB + PUD } & 17 & 0.29 & 0.30 & 0.33 & 0.31 \\
\hline & & $(0.21,0.38)$ & $\begin{array}{l}(0.20 \\
0.30)\end{array}$ & $(0.24,0.41)$ & $(0.30,0.35)$ \\
\hline \multirow[t]{2}{*}{ No acute UGIB + varices } & 66 & 0.33 & 0.30 & 0.38 & 0.31 \\
\hline & & $(0.28,0.37)$ & $\begin{array}{l}(0.20 \\
0.35)\end{array}$ & $(0.31,0.41)$ & $(0.31,0.35)$ \\
\hline \multirow[t]{2}{*}{ Acute UGIB + PUD } & 4 & 0.58 & 0.60 & 0.45 & 0.40 \\
\hline & & $(0.38,0.78)$ & $\begin{array}{l}(0.40, \\
0.70)\end{array}$ & $(0.19,0.70)$ & $(0.31,0.68)$ \\
\hline \multirow[t]{2}{*}{ Acute UGIB + varices } & 18 & 0.63 & 0.60 & 0.52 & 0.48 \\
\hline & & $(0.54,0.73)$ & $\begin{array}{l}(0.50, \\
0.70)\end{array}$ & $(0.41,0.63)$ & $(0.37,0.73)$ \\
\hline \multicolumn{6}{|l|}{ Liver fibrosis ultrasound patterns } \\
\hline \multirow[t]{2}{*}{ A(No fibrosis) } & 4 & 0.28 & 0.30 & 0.29 & 0.31 \\
\hline & & $(0.19,0.36)$ & $\begin{array}{l}(0.20 \\
, 0.30)\end{array}$ & $(0.20,0.39)$ & $(0.20,0.35)$ \\
\hline \multirow[t]{2}{*}{ C( mild periportal fibrosis) } & 5 & 0.35 & 0.40 & 0.41 & 0.35 \\
\hline & & $(0.15,0.57)$ & $\begin{array}{l}(0.10 \\
0.50)\end{array}$ & $(0.14,0.68)$ & $(0.20,0.78)$ \\
\hline \multirow[t]{2}{*}{$\mathrm{D}$ ( moderate periportal fibrosis) } & 34 & 0.35 & 0.30 & 0.37 & 0.31 \\
\hline & & $(0.27,0.42)$ & $\begin{array}{l}(0.20 \\
0.40)\end{array}$ & $(0.28,0.47)$ & $(0.30,0.35)$ \\
\hline
\end{tabular}

UGIB - upper gastrointestinal bleeding, BMI - body mass index, WHO-world health organization. 


\begin{tabular}{|c|c|c|c|c|c|}
\hline & & EQVAS-DW & $\begin{array}{l}\text { EQVAS- } \\
\text { DW }\end{array}$ & $\begin{array}{l}\text { EQ5D } \\
\text { index-DW }\end{array}$ & $\begin{array}{l}\text { EQ5D } \\
\text { index-DW }\end{array}$ \\
\hline \multirow[t]{2}{*}{ E ( Severe periportal fibrosis) } & 21 & 0.41 & 0.30 & 0.37 & 0.32 \\
\hline & & $(0.30,0.52)$ & $\begin{array}{l}(0.25 \\
, 0.50)\end{array}$ & $(0.31,0.44)$ & $(0.31,0.40)$ \\
\hline \multirow[t]{2}{*}{ F ( Severe periportal fibrosis) } & 3 & 0.40 & 0.50 & 0.36 & 0.36 \\
\hline & & $(0.30,0.83)$ & $\begin{array}{l}(0.20 \\
0.50)\end{array}$ & $(0.23,0.49)$ & $(0.31,0.40)$ \\
\hline \multirow[t]{2}{*}{ X ( Liver cirrhosis like picture) } & 38 & 0.42 & 0.40 & 0.41 & 0.35 \\
\hline & & $(0.34,0.49)$ & $\begin{array}{l}(0.30 \\
0.50)\end{array}$ & $(0.33,0.48)$ & $(0.31,0.41)$ \\
\hline \multicolumn{6}{|l|}{ Ascites ultrasound } \\
\hline \multirow[t]{2}{*}{ Yes } & 14 & 0.55 & 0.48 & 0.58 & 0.60 \\
\hline & & $(0.43,0.67)$ & $\begin{array}{l}(0.40- \\
0.74)\end{array}$ & $(0.49,0.67)$ & $(0.5,0.70)$ \\
\hline \multirow[t]{2}{*}{ No } & 91 & 0.36 & 0.31 & 0.35 & 0.3 \\
\hline & & $(0.32,0.40)$ & $\begin{array}{l}(0.31 \\
0.35)\end{array}$ & $(0.31,0.40)$ & $(0.30,0.40)$ \\
\hline
\end{tabular}


Table 3

Multivariable analysis of factors associated with the derived disability weights

\begin{tabular}{|c|c|c|}
\hline & Best & Best \\
\hline & VAS-DW & EQ5D-DW \\
\hline & model & model \\
\hline & b (se) & b (se) \\
\hline Age( years) & $0.004 * \star$ & $0.005^{\star \star}$ \\
\hline & $(0)$ & $(0)$ \\
\hline Ascites & $0.117 *$ & $0.128^{\star}$ \\
\hline & $(-0.04)$ & $(-0.05)$ \\
\hline Female & $0.069 *$ & $0.122^{\star \star}$ \\
\hline & $(-0.03)$ & $(-0.04)$ \\
\hline Acute UGIB & $0.276^{\star \star \star}$ & 0.130 ** \\
\hline & $(-0.04)$ & $(-0.04)$ \\
\hline constant & 0.084 & 0.048 \\
\hline & $(-0.07)$ & $(-0.08)$ \\
\hline r2 & 0.443 & 0.274 \\
\hline$d f \_r$ & 100 & 100 \\
\hline bic & -65.903 & -40.553 \\
\hline * $\mathrm{p}<0.05$, ** & $<0.001$ & \\
\hline $\begin{array}{l}\text { b- beta coeffi } \\
\text { df_r - residu }\end{array}$ & $\begin{array}{l}\text { andard err } \\
\text { f freedom, }\end{array}$ & $\begin{array}{l}\text { quared, } \\
\text { an information criteria }\end{array}$ \\
\hline UGIB- uppe & al bleedi & \\
\hline
\end{tabular}

\section{Discussion}

Our study assessed health-related quality of life measures among adult patients with UGIB at a rural primary health care facility in sub-Saharan Africa where S.mansoni is endemic. To the best of our knowledge, no other study has described similar findings from rural sub-Saharan Africa. Our study participants were at the highest risk of schistosomiasis because of frequent contact with the waters of the River Nile. Ninety seven percent had moderate to severe periportal fibrosis, $90 \%$ had splenomegaly, and $80 \%$ varices. This clinical profile is in keeping with a diagnosis of hepatosplenic schistosomiasis[20]. 
The differential diagnosis of such a profile is liver cirrhosis. This was less likely because of the low prevalence of alcohol misuse and viral hepatitis B or C infections. We also found a low prevalence of stigmata of chronic liver disease including ascites, hepatic encephalopathy, and jaundice. These stigmata are more frequent in liver cirrhosis than hepatosplenic schistosomiasis[21]. Peptic ulcer disease was infrequent and was less likely to be the cause of UGIB among our participants.

Prior to enrollment, none of our participants had endoscopy and no participant with varices was on propranolol for prevention of recurrent variceal bleeding. Current care guides recommend endoscopy for all with UGIB and/or propranolol for those with UGIB and varices[22-24].

Evaluation on EQ-5D primary data showed our participants reported more problems in all the five measured health domains (mobility, self-care, usual activities, pain/discomfort, and anxiety/depression) than one comparable HRQOL study on hepatic schistosomiasis from brazil. In addition, the mean and median VAS score from our study were also lower than the same HRQOL study from brazil[7]. These differences were greatest for the domains of welfare and anxiety/depression. We think this could be explained by population differences in disease severity (all our participants experienced UGIB, which is considered a severe disease) or differences in quality of health care services between the two study populations. On the other hand, our EQ-5D estimates (index and VAS) are similar to what has been reported by a systematic review on health state estimates among patients with advanced chronic liver disease[25]. This upholds our earlier assertion that our participants had severe liver disease.

From the transformed EQ-VAS and EQ5D index measurements, the average disability weights in our study population were found to be between 0.3 to 0.43 . Average disability weights for hematemesis alone were between $0.20-0.44$. These are similar to what has been published by the "Global Burden of Disease Study 2017 " that reported disability weights of between 0.21 to 0.46 for hematemesis and schistosomiasis [26, 27]. Other disability weights reported by the same study, "ascites and schistosomiasis" and "anemia and schistosomiases" were 4 to 5 times lower than what we found. On the other hand, the "Global Burden of Disease Study 2017" did not provide disability weights for acute UGIB. Simply put, our finding supports the ongoing argument that the "Global Burden of Disease Study 2017" probably underestimated the health impact of schistosomiasis[14].

The highest disability weights in our study were found among those who had all the three symptoms of UGIB (hematemesis, melena, and hematochezia) at enrollment, acute UGIB, acute UGIB with varices, ascites, and edema. We determined from multivariable analysis that older age, female sex, ascites, and acute UGIB were associated with the greatest probability of higher disability weights among those with UGIB and schistosomiasis. Similar findings have been reported by researchers from brazil and china for the factors age, female sex, and ascites[7, 12]. To the best of our knowledge, no study has directly linked the occurrence of acute UGIB and schistosomiasis to higher disability. What is known is that acute UGIB is the main reason for hospitalization and death among patients with schistosomiasis due to S.mansoni $[3,4,28,29]$. It therefore stands to reason that preventing acute UGIB could probably decrease health loss in this population[8]. In the same way, addressing ascites in this population could also decrease health 
loss. This assertion is supported by published research which shows many acute illnesses requiring hospitalization are usually associated with increased disability. Preventing hospitalization decreases disability or health loss[30].

Our study has limitations. These are related to our cross-sectional study design, the use of the measurement tool (EuroQol EQ-5D/EQ-VAS), and our relatively small study sample size. Although the EuroQol EQ-5D/ EQ-VAS is a generic tool, its use in schistosomiasis or UGIB worldwide is limited. As such, more studies are required to confirm our findings.

Nevertheless, our study provides new HRQOL measurements from rural Africa. These measurements are important in defining the different health states among patients with UGIB and schistosomiasis. The study also provides credible disability weights that are crucial for estimation of the global burden of disease and for health policy.

\section{Conclusions}

Adult patients with upper gastrointestinal bleeding from this part of rural Sub-Saharan Africa where S. mansoni is endemic frequently have varices, portal hypertension, splenomegaly, and ultrasound evidence of liver disease (periportal fibrosis and/or cirrhosis). Our study participants at this primary health facility experienced poor health. This was suggested by the large proportion of participants admitted (past or present) for UGIB, low health utility estimates, and relatively high disability weights. Older age, female gender, those with ascites or acute UGIB had the highest disability weights, and the greatest probability of having higher disability weights or the lowest health-related quality of life. These findings are new, unique, and improve the definitions of different health states among patients with UGIB and schistosomiasis. Lastly, our findings highlight the pressing need for primary, secondary, and tertiary prevention of ascites and acute UGIB in this patient population.

\section{Declarations}

\section{Consent for publication}

The authors provide formal consent to publish this Work.

\section{Availability of data and materials}

Data is available and is provided as supplementary material.

\section{Competing interests}

The authors declare that they have no competing interests. 


\section{Financial support}

The study was funded through an educational research grant from the Programmatic Award: Medical Education for Services to All Ugandans (MESAU). http://www.fic.nih.gov/Grants/Search/Pages/MEPIR24TW008886.aspx

\section{Acknowledgements}

The study was funded through an educational research grant from the Programmatic Award: Medical Education for Services to All Ugandans (MESAU). http://www.fic.nih.gov/Grants/Search/Pages/MEPIR24TW008886.aspx

KReLL family for providing the endoscope tower.

\section{Author contributions}

Protocol development : CKO, PO, FZ, LR

Data collection : CKO, PO, FZ, LR

Data analysis : CKO, PO, FZ, LR,NBK

Formulation of Manuscript: CKO, PO, FZ, LR, NBK

\section{References}

1. Alema ON, Martin DO, Okello TR. Endoscopic findings in upper gastrointestinal bleeding patients at Lacor hospital, northern Uganda. African health sciences. 2012;12:518-21.

2. Moledina SM, Komba E. Risk factors for mortality among patients admitted with upper gastrointestinal bleeding at a tertiary hospital: a prospective cohort study. BMC gastroenterology. 2017;17:165.

3. Chofle AA, Jaka H, Koy M, Smart LR, Kabangila R, Ewings FM, et al. Oesophageal varices, schistosomiasis, and mortality among patients admitted with haematemesis in Mwanza, Tanzania: a prospective cohort study. BMC Infect Dis. 2014;14:303.

4. Mulima G, Qureshi JS, Shores C, Tamimi S, Klackenberg H, Andrén-Sandberg Å. Upper Gastrointestinal Bleeding at a Public Referal Hospital in Malawi. Surgical Science. 2014;5:501.

5. Organization WH. Progress in assessment of morbidity due to Schistosoma mansoni infection: a review of recent literature. Geneva: World Health Organization; 1988.

6. Mohammed SEA, Abdo AE, Mudawi HMY. Mortality and rebleeding following variceal haemorrhage in liver cirrhosis and periportal fibrosis. World journal of hepatology. 2016;8:1336. 
7. Nascimento GL, Domingues ALC, Ximenes RA de A, Itria A, Cruz LN, Oliveira MRF de. Quality of life and quality-adjusted life years of chronic schistosomiasis mansoni patients in Brazil in 2015. Trans R Soc Trop Med Hyg. 2018;112:238-44.

8. Gunda DW, Kilonzo SB, Manyiri PM, Peck RN, Mazigo HD. Morbidity and Mortality Due to Schistosoma mansoni Related Periportal Fibrosis: Could Early Diagnosis of Varices Improve the Outcome Following Available Treatment Modalities in Sub Saharan Africa? A Scoping Review. Tropical Medicine and Infectious Disease [Internet]. Multidisciplinary Digital Publishing Institute; 2020 [cited 2020 Apr 12];5:20. Available from: https://www.mdpi.com/2414-6366/5/1/20

9. Opio CK, Garcia-Tsao G. Managing varices: drugs, bands, and shunts. Gastroenterology Clinics. 2011;40:561-79.

10. Litwin MS. Health-related quality of life. Clinical research methods for surgeons. Springer; 2006. p. 237-51.

11. Muragundi P, Tumkur A, Shetty R, Naik A. Health-related Quality of Life Measurement. J Young Pharm [Internet]. 2012 [cited 2020 Apr 30];4:54. Available from:

https://www.ncbi.nlm.nih.gov/pmc/articles/PMC3326784/

12. Jia T-W, Utzinger J, Deng Y, Yang K, Li Y-Y, Zhu J-H, et al. Quantifying the quality of life and disability of patients with advanced schistosomiasis japonica. PLoS neglected tropical diseases. Public Library of Science; 2011;5.

13. Nascimento GL, de Oliveira MRF. Severe forms of schistosomiasis mansoni: epidemiologic and economic impact in Brazil, 2010. Trans R Soc Trop Med Hyg. 2014;108:29-36.

14. King $\mathrm{CH}$, Galvani AP. Underestimation of the global burden of schistosomiasis. The Lancet. 2018;391:307-8.

15. Opio CK, Kazibwe F, Ocama P, Rejani L, Belousova EN, Ajal P. Profiling lifetime episodes of upper gastrointestinal bleeding among patients from rural Sub-Saharan Africa where schistosoma mansoni is endemic. The Pan African medical journal. 2016;24.

16. Opio CK, Rejani L, Kazibwe F, Ocama P. The diagnostic accuracy of routine clinical findings for detection of esophageal varices in rural sub-Saharan Africa where schistosomiasis is endemic. African Health Sciences. Makerere University Medical School (Uganda); 2019;19:3225-34.

17. Wille N, Badia X, Bonsel G, Burström K, Cavrini G, Devlin N, et al. Development of EQ-5D-Y: a childfriendly version of the EQ-5D. Quality of life research. Springer; 2010;19:875-86.

18. Devlin NJ, Brooks R. EQ-5D and EuroQol group: past, present, and future. Applied health economics and health policy. Springer; 2017;15:127-37.

19. Haagsma JA, Polinder S, Cassini A, Colzani E, Havelaar AH. Review of disability weight studies: comparison of methodological choices and values. Population health metrics. BioMed Central; 2014;12:20.

20. Lambertucci JR. Revisiting the concept of hepatosplenic schistosomiasis and its challenges using traditional and new tools. Revista da Sociedade Brasileira de Medicina Tropical. 2014;47:130-6. 
21. Rebouças G. Clinical aspects of hepatosplenic schistosomiasis: a contrast with cirrhosis. The Yale journal of biology and medicine. 1975;48:369.

22. Chen P-H, Chen W-C, Hou M-C, Liu T-T, Chang C-J, Liao W-C, et al. Delayed endoscopy increases rebleeding and mortality in patients with hematemesis and active esophageal variceal bleeding: a cohort study. Journal of hepatology. 2012;57:1207-13.

23. Kiire CF. Controlled trial of propranolol to prevent recurrent variceal bleeding in patients with noncirrhotic portal fibrosis. BMJ. 1989;298:1363-5.

24. Tourabi HE, Amin AE, Shaheen M, Woda SA, Homeida M, Harron DWG. Propranolol reduces mortality in patients with portal hypertension secondary to schistosomiasis. Annals of Tropical Medicine \& Parasitology. 1994;88:493-500.

25. Møller AH, Erntoft S, Vinding GR, Jemec GB. A systematic literature review to compare quality of life in psoriasis with other chronic diseases using EQ-5D-derived utility values. Patient related outcome measures. Dove Press; 2015;6:167.

26. Global Burden of Disease Study 2017 (GBD 2017) Disability Weights | GHDx [Internet]. [cited 2020 Apr 8]. Available from: http://ghdx.healthdata.org/record/ihme-data/gbd-2017-disability-weights

27. Vos T, Abajobir AA, Abate KH, Abbafati C, Abbas KM, Abd-Allah F, et al. Global, regional, and national incidence, prevalence, and years lived with disability for 328 diseases and injuries for 195 countries, 1990-2016: a systematic analysis for the Global Burden of Disease Study 2016. The Lancet. 2017;390:1211-59.

28. Barbosa CS, de Souza Gomes EC, Campos JV, de Oliveira FJM, da Silva Mesquita MC, de Oliveira ECA, et al. Morbidity of mansoni schistosomiasis in Pernambuco-Brazil: Analysis on the temporal evolution of deaths, hospital admissions and severe clinical forms (1999-2014). Acta Trop. 2016;164:10-6.

29. Kheir MM, Eltoum IA, Saad AM, Ali MM, Baraka OZ, Homeida MM. Mortality due to schistosomiasis mansoni: a field study in Sudan. Am J Trop Med Hyg. 1999;60:307-10.

30. Garland A, Metz LM, Bernstein CN, Peschken CA, Hitchon CA, Marrie RA. Hospitalization is associated with subsequent disability in multiple sclerosis. Multiple sclerosis and related disorders. Elsevier; 2017;14:23-8.

\section{Figures}




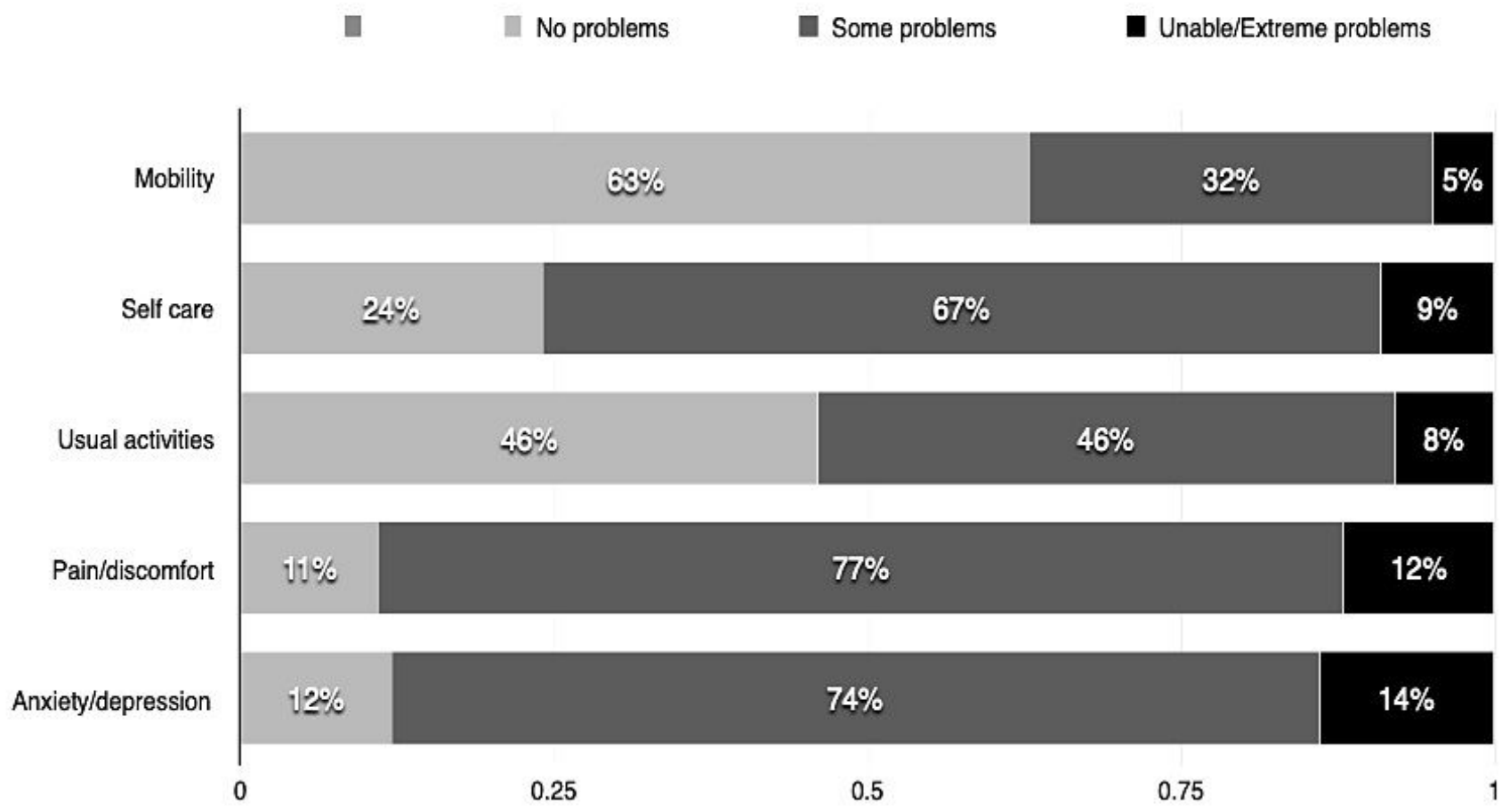

\section{Figure 1}

Frequency measures summarizing the 3 levels and 5 dimensions of health.

\section{Supplementary Files}

This is a list of supplementary files associated with this preprint. Click to download.

- EQ5DYUserGuide.pdf

- SUGIB2.xlsx 